\title{
Fusion of the Nucleoporin Gene, NUP98, and the Putative RNA Helicase Gene, DDX10, by Inversion 11 (p15q22) Chromosome Translocation in a Patient with Etoposide-related Myelodysplastic Syndrome
}

\author{
Katsuyuki NaKao, Maki Nishino, Kyoko TaKeuchi, Masayasu Iwata, Akira Kawano*, \\ Yasuhito ARAI** and Misao OHKI**
}

\begin{abstract}
We report a 50-year-old man who developed therapyrelated myelodysplastic syndrome after treatment with etoposide-including chemotherapy for extratesticular germ cell tumor. Chromosomal analysis showed inversion 11 (p15q22) translocation. Reverse transcriptase-polymerase chain reaction amplification of patient RNA showed a fusion transcript of nucleoporin gene $N U P 98$, and putative DEAD-box RNA helicase gene DDX10. NUP98 is implicated in the transformation through aberrant nucleocytoplasmic transport. $D D X 10$ is suggested to be involved in ribosome assembly. The NUP98-DDX10 fusion transcript may promote the development of secondary hematological malignancies caused by DNA-topoisomerase II inhibitors through aberrant nucleocytoplasmic transport and/or alteration in ribosome assembly.
\end{abstract}

(Internal Medicine 39: 412-415, 2000)

Key words: therapy-related myelodysplastic syndrome, secondary leukemia, fusion gene, NUP98/DDX10 fusion

\section{Introduction}

Therapy-related myelodysplastic syndrome (t-MDS) and therapy-related acute myeloid leukemia (t-AML) are the most feared long-term complications of cancer therapy. Several characteristic chromosomal aberrations have been reported in therapy-related hematological malignancies. Of these chromosomal aberrations, balanced translocations involving chromosome 11 are often observed after cytotoxic treatment including
DNA-topoisomerase II (topo II) inhibitors (1). One consequence of translocation is the production of chimeric fusion proteins. Recently, the putative DEAD-box RNA helicase gene $D D X 10$ has been reported to be rearranged and fused to the nucleoporin gene NUP98 in two inversion 11 (p15q22) translocation [inv(11) (p15q22)] patients who had received topo II inhibitor-including chemotherapy for primary tumors (2). We report here another etoposide-related MDS patient with the inv(11) (p15q22) who has a fusion transcript of NUP98 and $D D X 10$. We suggest that the NUP98-DDX10 fusion transcript is involved in the transformation caused by topo II inhibitors.

\section{Case Report}

A 50-year-old man visited our hospital because of macrohematuria in November 1994. The abdominal CT scan demonstrated retroperitoneal tumors. Biopsy showed a extratesticular germ cell tumor. Staging evaluation showed lung and liver metastases. He was treated with cytoreductive surgery and five cycles of chemotherapy including etoposide and cisplatin. The total cumulative dose of etoposide was 2,400 mg. Restaging in May 1995 revealed no evidence of disease. He remained well until January 1996, when he presented with fatigue and weakness. Physical examination showed hepatomegaly and splenomegaly. Peripheral blood showed a hemoglobin of $10.7 \mathrm{~g} / \mathrm{dl}$, a platelet count of $45 \times 10^{9} / l$, and a leukocyte count of $16.4 \times 10^{9} / l$ with $0 \%$ blasts and $40 \%$ mature monocytes. The monocyte count was $6.6 \times 10^{9} / l$. Bone marrow examination showed hypercellularity with $20 \%$ blasts (Fig. 1). Immunophenotypic markers of the blasts were CD13, CD33, CD36, and HLA-DR. Chromosomal analyses of bone marrow cells and peripheral blood cells showed 46, XY, inv(11) (p15q22) (Fig. 2). Serum levels of aspartate aminotransferase, alanine aminotransferase, alkaline phosphatase, total bilirubin,

From the Department of Internal Medicine, *the Department of Urology, Okawa General Hospital, Kagawa and **the Radiobiology Division, National Cancer Center Research Institute, Tokyo

Received for publication July 23, 1999; Accepted for publication November 11, 1999

Reprint requests should be addressed to Dr. Katsuyuki Nakao, the Department of Internal Medicine, Okawa General Hospital, 387 Ishidahigashi, Sangawa-cho, Okawa-gun, Kagawa 769-2393 
blood urea nitrogen, creatinine, and electrolytes were normal. Serum lactate dehydrogenase was slightly elevated to $220 \mathrm{IU} / \mathrm{l}$. Coagulation tests were within normal limits. Serum lysozyme and urinary lysozyme were elevated to $55 \mu \mathrm{g} / \mathrm{ml}$ and $1,170 \mu \mathrm{g} /$ $\mathrm{ml}$, respectively. He was diagnosed as chronic myelomonocytic leukemia $(\mathrm{CMMoL})$ according to the French-American-British classification (3). He was treated with oral administration of $100 \mathrm{mg} /$ day of cytarabine ocfosfate from March 1996. Leukocyte counts fluctuated between $10 \times 10^{9} / l$ and $40 \times 10^{9} / l$ with 40 to $60 \%$ monocytes. No blasts were found in peripheral blood.

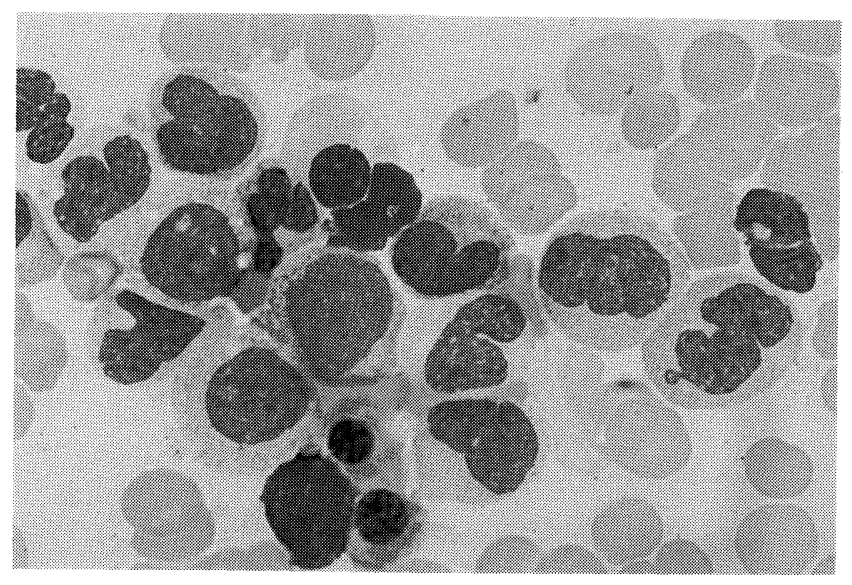

Figure 1. Light microscopic examination of bone marrow cells showing blasts and monocytic lineage cells (Wright-Giemsa stain, $\times 330$ ).
Bone marrow blasts represented less than $30 \%$ of hematopoietic cells. In February 1997, peripheral blood showed a leukocyte count of $33.2 \times 10^{9} / l$ with $61 \%$ blasts. Thus, evolution to acute leukemia occured.

To detect NUP98-DDX10 fusion transcript, we carried out reverse transcriptase-polymerase chain reaction (RT-PCR) amplification using NUP98 and DDX10 sequence specific primers with RNA from patient peripheral blood mononuclear cells in the MDS phase. RT-PCR was performed according to previously described methods (2). A 505-bp PCR product of NUP98$D D X 10$ fusion transcript was generated from the patient's RNA but not from control RNA (Fig. 3).

\section{Discussion}

We reported a patient who was successfully treated for a germ cell tumor with a regimen including etoposide and cisplatin and who subsequently developed CMMoL eight months later. Pedersen-Bjergaard et al (4) described that etoposide alone or in combination with cisplatin increases the risk of secondary leukemia. Because our patient has been exposed to multiple cytotoxic agents, etoposide cannot be implicated to be the causative agent of MDS. However, secondary hematological malignancies caused by etoposide have the following features: short latency period, monocytic or myelomonocytic feature, and chromosomal aberration including chromosome 11 (5). These features were also observed in the present patient. Thus, we diagnosed the patient as etoposiderelated MDS.

Balanced translocations involving chromosome bands 11q23
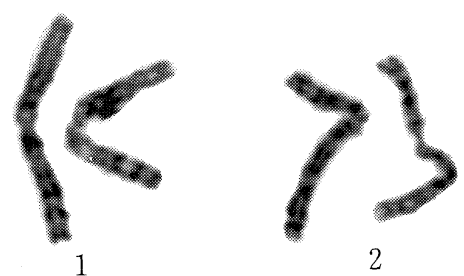
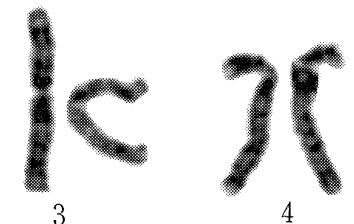

4

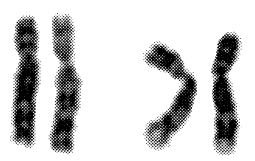

9

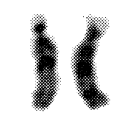

15

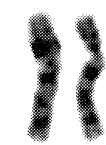

10

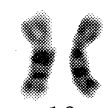

16

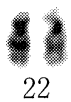

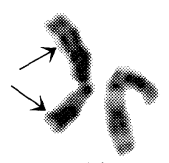

11
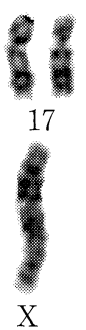

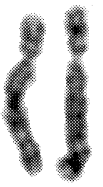

5

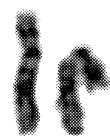

12

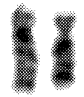

18

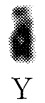

Figure 2. Cytogenetic analysis of bone marrow cells indicates $46, \mathrm{XY}$, inversion 11(p15q22)(arrows). 
NAKAO et al

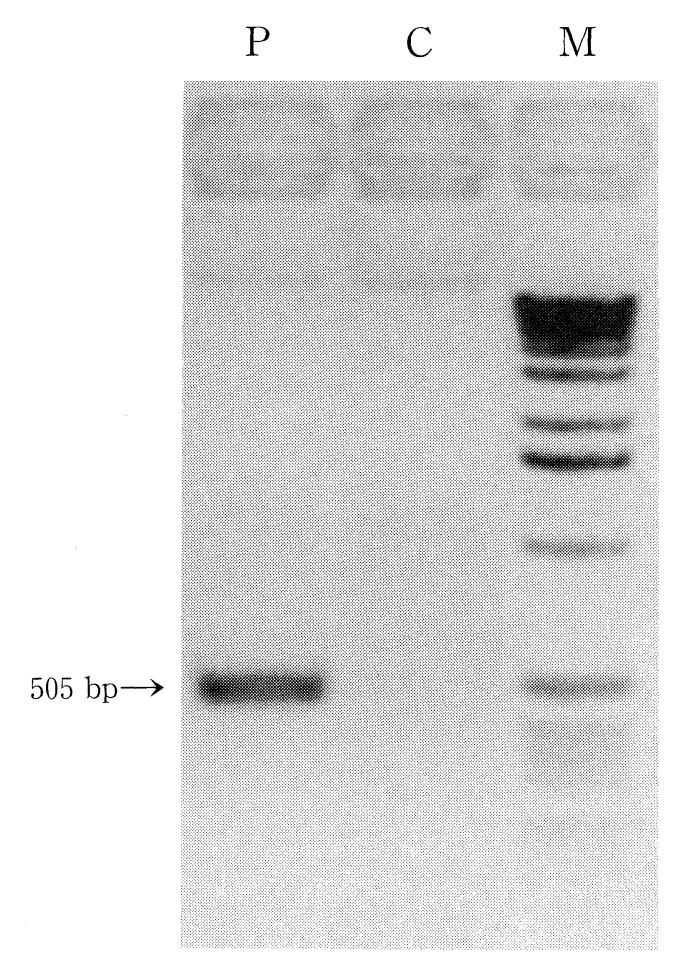

Figure 3. Detection of a fusion transcript between NUP98 and DDX10 by RT-PCR. P: RT-PCR amplified patient RNA sample. C: RT-PCR amplified normal peripheral blood RNA. M: Size marker. Arrow indicates the detected 505-bp fusion transcript.

and 21q22 in t-MDS or t-AML are definitely associated with previous therapy with topo II inhibitors (1). But, chromosomal analysis of our patient showed the inv(11) (p15q22). In in vitro study using phytohemagglutinin-stimulated normal human lymphocytes, Maraschin et al (6) demonstrated that chromosome band 11q22 tends to be rearranged after exposure to etoposide. This finding supports the proposal that the chromosomal aberrations involving $11 \mathrm{q} 22$ observed in t-MDS or t-AML are in fact primary and direct effects of an exposure of hematopoietic cells to etoposide. The inv (11) (p15q22) is observed rarely in de novo MDS and AML, but is found more frequently in tMDS and t-AML. Kobayashi et al (7) have reported that the inv (11) (p15q22) is seen in one of $371(0.3 \%)$ patients with de novo MDS or AML and in 2 of $11(18 \%)$ patients with t-MDS or t-AML induced by combination chemotherapy including topo II inhibitors. Thus, the inv (11) (p15q22), like 11q23 or 21q22 translocation, seems to be important in the pathogenesis of therapy-related hematological malignancies caused by topo II inhibitors.

Most of the translocations or inversions in leukemia have resulted in the formation of abnormal chimeric genes (1). Chimeric mRNAs between $M L L$ gene on 11q23 and various partner genes are formed in de novo or topo II inhibitor-related leukemias with 11q23 translocations (8). We detected a fusion transcript of NUP98 and DDX10 in our etoposide-related MDS patient. Recently, Arai et al (2) have reported that the putative DEAD-box RNA helicase gene DDX10 is rearranged in four inv (11) (p15q22) patients with de novo AML, de novo MDS, $\mathrm{t}-\mathrm{AML}$, and $\mathrm{t}-\mathrm{MDS}$. Moreover, they have demonstrated that $D D X 10$ is fused to the nucleoporin gene NUP98, resulting in NUP98-DDX10 fusion transcript, and that DDX10 and NUP98 are ubiquitously expressed. DDX10 is a novel human DEADbox RNA helicase gene on chromosome 11q22-q23 (9). DEADbox proteins comprise a family of putative ATP-dependent RNA helicases implicated in many cellular processes involving alteration of RNA secondary structure, such as translation initiation, nuclear and mitochondrial RNA splicing, ribosome and spliceosome assembly (10). DDX10, although its normal function has not yet been identified, is suggested to be involved in ribosome assembly because the predicted amino acid sequence of $D D X 10$ protein shows high similarity to a subgroup of DEAD-box proteins involved in ribosome biogenesis (9). NUP98 is a new nucleoporin gene identified on chromosome $11 \mathrm{p} 15(11,12)$. NUP98 protein is located at the nucleoplasmic side of the nuclear pore complex and promotes bidirectional transport of protein and RNA across the nuclear membrane (13). A possible role for NUP98 in transformation is suggested by the involvement of NUP98 in the $\mathrm{t}(7 ; 11)(\mathrm{p} 15 ; \mathrm{p} 15)$ translocation associated with AML $(11,12)$. The $t(7 ; 11)(\mathrm{p} 15 ; \mathrm{p} 15)$ translocation fuses NUP98 to a class I homeobox gene HOXA9, suggesting that aberrant nucleocytoplasmic transport can contribute to transformation.

Therefore, we suggest that the NUP98-DDX10 fusion transcript is involved in transformation induced by topo II inhibitors through aberrant nucleocytoplasmic transport and/or alteration in ribosome assembly. Whether the inv (11) (p15q22) disrupts both NUP98-mediated nucleocytoplasmic transport and DDX10-mediated ribosome assembly, or whether one protein serves only to modulate the activity of the other is presently unclear. Further study with more patients is necessary to clarify the correlation between the NUP98-DDX10 fusion transcript and leukemogenesis.

\section{References}

1) Pedersen-Bjergaard J, Rowley JD. The balanced and the unbalanced chromosome aberrations of acute myeloid leukemia may develop in different ways and may contribute differently to malignant transformaton. Blood 83: 2780-2786, 1994.

2) Arai Y, Hosoda F, Kobayashi H, et al. The inv(11)(p15q22) chromosome translocation of de novo and therapy-related myeloid malignancies results in fusion of the nucleoporin gene, NUP98, with the putative RNA helicase gene, DDX10. Blood 89: 3936-3944, 1997.

3) Bennett JM, Catovsky D, Daniel MT, et al. Proposals for the classification of the myelodysplastic syndromes. Br J Haematol 51: 189-199, 1982.

4) Pedersen-Bjergaard J, Daugaard G, Hansen SW, et al. Increased risk of myelodysplasia and leukaemia after etoposide, cisplatin, and bleomycin for germ-cell tumours. Lancet 338: 359-363, 1991.

5) Whitlock JA, Greer JP, Lukens JN. Epipodophyllotoxin-related leukemia: Identification of a new subset of secondary leukemia. Cancer 68: 600-604, 1991.

6) Maraschin J, Dutrillaux B, Aurias A. Chromosome aberrations induced by etoposide (VP-16) are not random. Int J Cancer 46: 808-812, 1990.

7) Kobayashi $\mathrm{H}$, Arai $\mathrm{Y}$, Hosoda F, et al. Inversion of chromosome 11, inv 


\section{NUP98-DDX10 Fusion Gene in t-MDS}

(11) (p15q22), as a recurring chromosomal aberration associated with de novo and secondary myeloid malignancies: Identification of a P1 clone spanning the 11q22 breakpoint. Genes Chromosom Cancer 19: 150-155, 1997.

8) Yamamoto $K$, Seto $M$, Iida $S$, et al. A reverse transcriptase-polymerase chain reaction detects heterogeneous chimeric mRNAs in leukemias with 11q23 abnormalities. Blood 83: 2912-2921, 1994.

9) Savitsky K, Ziv Y, Bar-Shira A, et al. A human gene (DDX10) encoding a putative DEAD-box RNA helicase at 11q22-q23. Genomics 33: 199206, 1996.

10) Schmid SR, Linder P. D-E-A-D protein family of putative RNA helicases.
Mol Microbiol 6: 283-292, 1992.

11) Nakamura T, Largaespada DA, Lee MP, et al. Fusion of the nucleoporin gene NUP98 to HOXA9 by the chromosome translocation $t(7 ; 11)$ (p15;p15) in human myeloid leukaemia. Nature Genet 12: 154-158, 1996.

12) Borrow J, Shearman AM, Stanton Jr VP, et al. The t $(7 ; 11)$ (p15;p15) translocation in acute myeloid leukaemia fuses the genes for nucleoporin NUP98 and class I homeoprotein HOXA9. Nature Genet 12: 159-167, 1996.

13) Radu A, Moore MS, Blobel G. The peptide repeat domain of nucleoporin Nup98 functions as a docking site in transport across the nuclear pore complex. Cell 81: 215-222, 1995. 